\title{
A randomised, investigator-initiated, clinical trial of the effects of fentanyl on P2Y12-receptor inhibition in patients with ST-elevation myocardial infarction who are pre-treated with crushed ticagrelor: rationale and design of the Opioids aNd crushed Ticagrelor In Myocardial infarction Evaluation (ON-TIME 3) trial
}

\author{
A. H. Tavenier · R. S. Hermanides - J. P. Ottervanger · S. Rasoul · R. J. Slingerland · R. Tolsma $\cdot$ S. van Workum $\cdot$ \\ E. Kedhi · A. W. J. van 't Hof
}

Published online: 14 February 2019

(C) The Author(s) 2019

\begin{abstract}
Background Fast and accurate platelet inhibition is an important therapeutic goal in the acute treatment of patients with ST-elevation myocardial infarction (STEMI). Platelet inhibitory effects induced by oral P2Y12-receptor antagonists are delayed in STEMI patients undergoing primary percutaneous coronary intervention (PCI) due to haemodynamic changes and delayed gastro-intestinal absorption. Concomitant use of opioids, although recommended in the American College of Cardiology/American Heart Association and European Society of Cardiology STEMI guidelines, further delays gastro-intestinal absorption. To date, trials investigating alternative analgesics in STEMI patients have been scarce. This trial aims to assess the feasibility of a novel drug strategy for
\end{abstract}

\footnotetext{
A. H. Tavenier $\cdot$ R. S. Hermanides $(\bowtie) \cdot$ J. P. Ottervanger E. Kedhi

Department of Cardiology, Isala, Zwolle, The Netherlands r.s.hermanides@isala.nl

S. Rasoul · A. W. J. van 't Hof

Department of Cardiology, Maastricht University Medical Centre, Maastricht, The Netherlands

Department of Cardiology, Zuyderland Medical Centre, Heerlen, The Netherlands

R. J. Slingerland

Department of Clinical Chemistry, Isala, Zwolle, The Netherlands

R. Tolsma

RAV IJsselland, Zwolle, The Netherlands

S. van Workum

RAV Zuid-Limburg, Heerlen, The Netherlands
}

treatment of STEMI patients with crushed ticagrelor in combination with paracetamol (acetaminophen) instead of opioids.

Hypothesis STEMI patients who are pre-treated with crushed ticagrelor and paracetamol have a higher level of platelet inhibition after primary PCI than patients pre-treated with crushed ticagrelor and fentanyl.

Study design The Opioids aNd crushed Ticagrelor In Myocardial infarction Evaluation (ON-TIME 3) trial is a randomised controlled trial designed to examine whether administration of paracetamol instead of fentanyl can optimise platelet inhibition in STEMI patients who are pre-treated with crushed ticagrelor in the ambulance. One hundred and ninety patients with STEMI will be randomised (1:1 fashion) to intravenous (IV) fentanyl or IV paracetamol. The primary endpoint is the level of platelet reactivity units measured immediately after primary PCI.

Summary The ON-TIME 3 trial (NCT03400267) aims to achieve optimal platelet inhibition and pain relief in STEMI patients receiving crushed ticagrelor in the ambulance by investigating IV fentanyl and IV paracetamol as analgesics.

Keywords ST-elevation myocardial infarction - Ticagrelor · Platelet inhibition - Primary percutaneous coronary intervention · Fentanyl

\section{Background}

Adequate and optimal platelet inhibition is an important therapeutic goal in the treatment of patients with ST-elevation myocardial infarction (STEMI). 
Platelet inhibitory effects induced by potent oral P2Y12-receptor antagonists like ticagrelor are delayed in STEMI patients undergoing primary percutaneous coronary intervention (PCI) [1]. STEMI-induced selective shunting of blood to vital organs decreases gastro-intestinal perfusion and induces impaired drug absorption. Furthermore, analgesic drugs such as fentanyl and morphine, which are recommended according to American College of Cardiology/American Heart Association (ACC/AHA) and European Society of Cardiology (ESC) STEMI guidelines [2, 3], are used in STEMI to reduce pain-associated sympathetic activation, which increases vasoconstriction, blood pressure and heart rate [4]. In addition, the uptake of P2Y12 inhibitors can be further delayed by drug interactions. In particular, morphine delays the intestinal drug absorption of P2Y12 inhibitors [5].

In the past, different trials have investigated the influence of morphine on platelet inhibition. The IMPRESSION trial [5] found that administration of morphine in patients with STEMI and non-STEMI influenced the pharmacokinetics by lowering the total exposure to in-hospital-administered ticagrelor and its active metabolite by $36 \%$ and $37 \%$, respectively, and delayed the time to maximal plasma concentration of ticagrelor. Also, the effects of morphine on pharmacodynamics have been described and, more frequently, a high platelet reactivity was observed in STEMI patients treated with a $\mathrm{P} 2 \mathrm{Y} 12$ inhibitor and receiving morphine $[5,6]$, which is a phenomenon known to be associated with acute stent thrombosis [7, 8]. The PACIFY trial [9] investigated the influence of fentanyl, a potent opioid, on platelet inhibition and showed lower plasma concentrations of ticagrelor and delayed antiplatelet effects in patients receiving fentanyl during elective angiography.

Different studies have shown that morphine may have adverse effects on inhibitory properties of antiplatelet agents [10-12], myocardial reperfusion [13, 14 , and also on all-cause mortality in patients with acute myocardial infarction [15]. However, reports from other studies failed to show such an association [16]. Finally, the pain-relieving effects of morphine in STEMI patients have not been fully investigated yet. To date, trials investigating alternative analgesics in STEMI patients have been scarce.

Different routes of administration of P2Y12 inhibitors like ticagrelor in STEMI patients have been investigated to improve gastro-intestinal absorption. The MOJITO study [17] randomised patients with STEMI undergoing primary PCI into crushed or integral tablets of ticagrelor. Crushed ticagrelor tablet administration in STEMI patients was feasible and provided earlier platelet inhibition compared with standard integral tablets. However, sublingual crushed administration of ticagrelor did not further improve the uptake in patients with unstable angina [18].
The Opioids aNd crushed Ticagrelor In Myocardial infarction Evaluation (ON-TIME 3) trial aims to study the impact of an alternative non-opioid analgesic, paracetamol (acetaminophen), as compared to fentanyl in combination with crushed ticagrelor administration in the ambulance on platelet inhibition in STEMI patients.

\section{Methods}

\section{Study design and objective}

The ON-TIME 3 trial is a prospective, randomised trial, of which the primary objective is to assess the level of platelet inhibition after primary PCI in STEMI patients who are pre-treated with crushed ticagrelor and paracetamol compared to patients who are pretreated with crushed ticagrelor and fentanyl. The study will be performed in two hospitals: Isala Hospital in Zwolle and Zuyderland Medical Centre in Heerlen. All study drugs are administered in the ambulance.

\section{Trial registration}

This study will be conducted in accordance with the Declaration of Helsinki (64th WMA General Assembly, Fortaleza, Brazil, October 2013), the Medicinal Research Involving Human Subjects Act (WMO) and ICH-Good Clinical Practice (GCP). The study protocol and patient informed consent are approved by the local ethics committee. The ON-TIME 3 trial is registered on ClinicalTrials.gov (NCT03400267).

\section{Study protocol, randomisation and follow-up}

All STEMI patients (defined as on-going chest pain $>30 \mathrm{~min}$ and $<12 \mathrm{~h}$ duration and ST-segment elevation $>0.1 \mathrm{mV}$ in at least two contiguous leads) as diagnosed by the paramedic team on arrival at the patient site that comply with the inclusion and exclusion criteria (Tab. 1) will be asked to participate in this trial. After verbal informed consent patients will be randomised in a 1:1 fashion to either intravenous (IV) fentanyl or IV paracetamol (Fig. 1). The trial is not blinded. All patients will be pre-loaded in the ambulance with unfractionated heparin 5,000 IU and aspirin $500 \mathrm{mg}$ IV according to standard care and $180 \mathrm{mg}$ crushed oral ticagrelor. The paramedic team will register vital parameters and pain scores on a 10-step pain scale. Descriptive statistics will be derived from electronic medical clinical records and stored in an electronic case report form (eCRF). Haemodynamic parameters and data on intensity of pain will be collected in the ambulance at initial presentation. Data on intensity of pain and data on platelet inhibition, including pharmacokinetics and pharmacodynamics, will be collected before (T1) and immediately after primary PCI (T2) at the catheterisation laboratory, $1 \mathrm{~h}$ 
Table 1 Inclusion and exclusion criteria

\begin{tabular}{|c|c|}
\hline Inclusion criteria & Exclusion criteria \\
\hline STEMI patients & $\begin{array}{l}\text { Presenting with cardiogenic shock; defined as: systolic blood pressure }<90 \mathrm{~mm} \mathrm{Hg} \text { and heart rate }>100 / \mathrm{min} \\
\text { and peripheral oxygen saturation }<90 \% \text { (without oxygen administration) }\end{array}$ \\
\hline Age $\geq 18$ years & Patient with a nasogastric tube in situ or requiring a nasogastric tube \\
\hline $\begin{array}{l}\text { Symptom onset to STEMI diagnosis ( }>30 \mathrm{~min} \\
\text { but }<12 \mathrm{~h} \text { ) }\end{array}$ & Patients who have already received fentanyl or paracetamol $<2 \mathrm{~h}$ prior to randomisation \\
\hline \multirow{7}{*}{$\begin{array}{l}\text { On-going chest pain with pain score } \geq 4 \text { (out of } \\
10 \text {-step pain score) }\end{array}$} & Patients on current treatment with P2Y12 inhibitors (ticagrelor, clopidogrel or prasugrel) \\
\hline & Patients allergic to fentanyl or paracetamol \\
\hline & $\begin{array}{l}\text { Patients with recent major bleeding complications or contraindication to dual antiplatelet therapy: hypersensi- } \\
\text { tivity to aspirin or ticagrelor, current use of (new) oral anticoagulation, history of bleeding diathesis or known } \\
\text { coagulopathy, active bleeding, refusal of blood transfusions, history of intracerebral mass, aneurysm, arteri- } \\
\text { ovenous malformation, or haemorrhagic stroke, and/or known severe liver dysfunction }\end{array}$ \\
\hline & Patients who have received any organ transplant or are on a waiting list for any organ transplant \\
\hline & Patients undergoing dialysis \\
\hline & Pregnant or lactating females \\
\hline & Patients currently participating in another investigational drug or device study \\
\hline
\end{tabular}

(T3), 3 and $6 \mathrm{~h}$ (T4) post-primary PCI at the coronary care unit. A 30-day post-randomisation followup will be performed by telephone interview (Fig. 1). Pharmacokinetics will be evaluated using appropriate pharmacokinetics modelling software (e.g. NONNEM or MWPharm) by determination of the concentration of ticagrelor and its active metabolite, AR-C124910XX, using liquid chromatography-mass spectrometry in the clinical chemistry laboratory in Zwolle. Pharmacodynamics will be assessed by a VerifyNow point of care test that measures the platelet reactivity units (PRU). Patients will be treated during hospital admission according to current ACC/AHA and ESC STEMI guidelines [2, 3]. Written informed consent will be obtained during hospital admission.

\section{Endpoints}

\section{Primary endpoint}

The primary endpoint of the study is the level of PRU measured immediately post-primary PCI or $1 \mathrm{~h}$ postangiography (Tab. 2). Blood will be obtained for our primary endpoint just before sheath removal (T2).

\section{Secondary endpoints}

The secondary endpoints (Tab. 2) consist of the pain reduction on a 10-step pain scale immediately post-primary PCI, the level of PRU at other time points, high on platelet reactivity (HPR) defined as PRU $>208$ at all time points, the area under the curve of the concentrations of ticagrelor and its active metabolite at all time points, and thrombolysis in myocardial infarction (TIMI) 3 flow in the culprit vessel after primary PCI. Exploratory safety endpoints are major adverse cardiac events, defined as cardiac death, non-fatal re-myocardial infarction or target vessel revascularisation, stent thrombosis (ARC criteria [19]), myocardial infarction after PCI [20], non-coronary-artery-bypass-grafting (CABG)-re- lated major bleeding (BARC 3 and 5 criteria [21]) and all-cause mortality at 30 days of follow-up.

\section{Study drugs}

Crushed ticagrelor ( $180 \mathrm{mg}$ ) will be prepared by using a crusher cup (Livsane), which allows preparation of crushed ticagrelor in an average time of $1-2 \mathrm{~min}$. After 2 times 5 rotations of the crushing mechanism, the crushed pill contents will be mixed with $25 \mathrm{ml}$ of $\mathrm{H}_{2} \mathrm{O}$ for $30 \mathrm{~s}$ in a $150-\mathrm{ml}$ dosing cup. The syringe crusher will be rinsed using an additional $25 \mathrm{ml}$ of $\mathrm{H}_{2} \mathrm{O}$ and will be added to the dosing cup for a total of $50 \mathrm{ml}$ suspension, which will be administered orally. When vomiting occurs and ticagrelor has been spit out, the patient will receive another loading dose of ticagrelor at the catheterisation laboratory. Paracetamol and fentanyl will be intravenously administered. Paracetamol $10 \mathrm{mg} / \mathrm{ml}$ is available in ampules of $100 \mathrm{ml}$ $(1000 \mathrm{mg})$ and its infusion time is $15 \mathrm{~min}$. Fentanyl $50 \mu \mathrm{g} / \mathrm{ml}$ is available in $2 \mathrm{ml}$ ampules $(100 \mu \mathrm{g})$ and will be titrated based on the weight of the patient. Its infusion time is $30 \mathrm{~s}$. The inhibitory pharmacodynamic or pharmacokinetic effects of paracetamol on platelet inhibition have not yet been described in the literature [22-24]. The use of all other intracoronary medication (heparin, nitroglycerin, adenosine and verapamil), the procedural technique at coronary angiography and the type of drug-eluting stent will be at the discretion of the operator. Administration of glycoprotein IIb/IIIa-receptor inhibitors will be restricted to bail-out situations only.

\section{Pre-specified subgroups for analysis}

If a patient randomised to the paracetamol arm experiences unbearable pain (score $>8$ on a 10-step pain scale), bail-out medication like fentanyl $50 \mu \mathrm{g}(1 \mathrm{ml})$ can be given. Patients with bail-out use of fentanyl 
Study drug

\section{Primary PCI}

Fentanyl

Paracetamol

T1

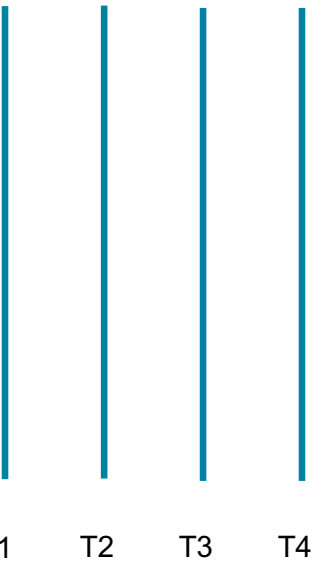

STEMI ST-elevation myocardial infarction, Primary $P C /$ primary percutaneous coronary intervention

Fig. 1 Schematic chart of ON-TIME 3 trial with randomisation and time of measurements ( $T 1$ arrival at cath lab, $T 2$ end of primary $\mathrm{PCl}, \mathrm{T} 31 \mathrm{~h}$ post-primary $\mathrm{PCl}, \mathrm{T} 46 \mathrm{~h}$ post-primary $\mathrm{PCl}$ ).

will be registered and analysed as a subgroup. Also patients with vomiting will be analysed as a subgroup.

\section{Statistical considerations}

This trial is powered for the primary endpoint. Analyses will be performed for both the intention-to-treat and per-protocol population. The primary endpoint will be based on an intention-to-treat analysis.

\section{Data analysis}

Continuous variables in both study arms will be compared using a Student's t-test or Mann-Whitney $U$ test depending on the presence or absence of a normal distribution of the data as assessed by the Kolmogorov-Smirnov test. Proportions will be compared by the Chi-square test or a Fisher's exact test when appropriate. Exploratory endpoints may be underpowered, and hence graphical methods and es- timates with $95 \%$ confidence intervals will be used for evaluation. An interim analysis at $50 \%$ of inclusions is planned to monitor efficacy and safety during study enrolment.

\section{Sample size calculation}

The ON-TIME 3 is a superiority trial assessing the use of IV paracetamol compared to IV fentanyl in STEMI patients. Since the effects of paracetamol on PRU are not known yet and no identical studies have been performed, an assumption of the sample size was necessary. We partly based our sample size calculation on data of the IMPRESSION trial [5] and PACIFY trial [9, 25]. Assuming a 60 PRU mean difference immediately after primary PCI between the two arms after a prehospital crushed ticagrelor loading dose with a standard deviation of 120 PRU, and $10 \%$ rate of invalid results due to haemolysis or technical problems, we

Table 2 Primary, secondary and exploratory endpoints

\begin{tabular}{|c|c|c|}
\hline & Secondary endpoint & Exploratory endpoints (at 30 days of follow-up) \\
\hline \multirow[t]{5}{*}{$\begin{array}{l}\text { Level of PRU measured immediately post-primary } \\
\mathrm{PCl} \text { or } 1 \mathrm{~h} \text { post-angiography (T2) }\end{array}$} & $\begin{array}{l}\text { Pain reduction on a 10-step pain scale immediately } \\
\text { post-primary PCI or } 1 \mathrm{~h} \text { post-angiography (T2) }\end{array}$ & $\begin{array}{l}\text { MACE (defined as cardiac death, non-fatal re-my- } \\
\text { ocardial infarction or target vessel revascularisa- } \\
\text { tion) }\end{array}$ \\
\hline & Level of PRU at other time points ( $\mathrm{T} 1, \mathrm{~T} 3, \mathrm{~T} 4)$ & Stent thrombosis (ARC criteria [19]) \\
\hline & $\begin{array}{l}\text { High on platelet reactivity defined as PRU }>208 \text { at } \\
\text { all time points (T1-4) }\end{array}$ & Myocardial infarction after PCI [20] \\
\hline & $\begin{array}{l}\text { Area under the curve of the concentrations of } \\
\text { ticagrelor and its active metabolite at all time } \\
\text { points (T1-4) }\end{array}$ & $\begin{array}{l}\text { Non-CABG-related major bleeding (BARC } 3 \text { and } 5 \\
\text { criteria [21]) }\end{array}$ \\
\hline & TIMI 3 flow in the culprit vessel after primary PCI & All-cause mortality \\
\hline
\end{tabular}


estimate that 190 patients (95 per arm) will be needed to obtain a $90 \%$ power and two-sided alpha of 0.05 .

\section{Expected results}

The ON-TIME 3 trial will be performed to show that STEMI patients treated pre-hospital with crushed ticagrelor and paracetamol have a higher level of platelet inhibition immediately after primary PCI than STEMI patients treated with crushed ticagrelor and fentanyl. The results of this trial have potential implications for the pre-hospital treatment of STEMI patients in the future.

\section{Discussion}

The absorption of more potent P2Y12 platelet inhibitors (ticagrelor and prasugrel) is delayed in STEMI patients due to a reduced gastric perfusion and impaired gastric emptying [1]. Opioids have widely been used in daily practice in STEMI patients and are recommended in international guidelines [2, 3], but opioids also seem to impair gastro-intestinal absorption by reducing gut motility and can further delay the onset of the effect of platelet inhibitors [5]. Moreover, nausea and vomiting are more often seen in patients receiving opioids $[4,6]$, which reduce the uptake of platelet inhibitors. Therefore, a non-opioid analgesic like paracetamol (acetaminophen) may be beneficial in this respect.

Before effective platelet inhibition is reached, time passes and a gap of sub-optimal platelet inhibition exists, which is an important predictor of ischaemic complications, such as stent thrombosis [7, 8]. To accelerate and improve the absorption of platelet inhibitors several possibilities have been investigated, like pre-hospital administration of oral platelet inhibitors, crushed or chewed ticagrelor and IV administration of platelet inhibitors [26-28]. Administration of crushed ticagrelor has been shown to reduce the time to effective platelet inhibition in STEMI patients compared with standard integral tablets [17].

Other analgesics might be an alternative for opioid use in STEMI patients. While non-steroidal anti-inflammatory drugs are known to increase cardiovascular events [29], paracetamol might be a suitable wellknown alternative. IV administered paracetamol is effective more quickly than its oral form. Just a few clinically relevant side effects are known. The main side effect is hypotension when administered intravenously [30]. However, gastro-intestinal side effects of paracetamol are less clear.

The ON-TIME 3 trial continues to search for pain relief and fast and optimal platelet inhibition by investigating an alternative analgesic, paracetamol IV, in STEMI patients that all received crushed ticagrelor in a pre-hospital setting. The results of this trial have potential implications for the pre-hospital treatment of STEMI patients in the future and may change ambulance guidelines.

\section{Limitations}

Although we carefully designed our study, some limitations are present. Firstly, the investigated treatment group is a paracetamol arm instead of a placebo arm. IV paracetamol and IV fentanyl are administered in different ways. Therefore, blinding for randomisation to IV paracetamol and IV fentanyl was not possible. Secondly, since the primary endpoint is the PRU value immediately after primary PCI, the number of patients in this trial is too small to test our hypothesis on clinical outcomes. Moreover, the follow-up period of this trial is only a month after randomisation and therefore long-term outcome and conclusions cannot be drawn based on this research, although optimal antiplatelet therapy in the first hours of onset of STEMI and treatment with primary PCI is thought to be essential for long-term outcomes.

\section{Summary}

The ON-TIME 3 trial is a prospective randomised trial which aims to assess whether STEMI patients who are treated pre-hospital with crushed ticagrelor and IV paracetamol have a higher level of platelet inhibition after primary PCI than patients treated with crushed ticagrelor and IV fentanyl.

Funding The study will be conducted with an unrestricted grant of AstraZeneca and an unrestricted grant of the Isala Academy. AstraZeneca and the Isala Academy reviewed the study protocol during its development and were allowed to make suggestions, but the final content was determined by the authors.

Conflict of interest The ON-TIME 3 trial is funded by AstraZeneca and the Isala Academy. Dr. van 't Hof reports institutional fees and non-financial support from AstraZeneca as well as grants from Medtronic. A.H. Tavenier, R.S. Hermanides, J.P. Ottervanger, S. Rasoul, R.J. Slingerland, R. Tolsma, S. van Workum and E. Kedhi declare that they have no competing interests.

Open Access This article is distributed under the terms of the Creative Commons Attribution 4.0 International License (http://creativecommons.org/licenses/by/4.0/), which permits unrestricted use, distribution, and reproduction in any medium, provided you give appropriate credit to the original author(s) and the source, provide a link to the Creative Commons license, and indicate if changes were made.

\section{References}

1. Heestermans AA, van Werkum JW, Taubert D, et al. Impaired bioavailability of clopidogrel in patients with a STsegment elevation myocardial infarction. Thromb Res. 2008;122(6):776-81.

2. O'Gara PT, Kushner FG, Ascheim DD, Casey DE Jr, et al. 2013 ACCF/AHA guideline for the management of ST-el- 
evation myocardial infarction: a report of the American College of Cardiology Foundation/American Heart Association Task Force on Practice Guidelines. Circulation. 2013;127(4):e362-425.

3. Ibanez B, James S, Agewall S, et al. 2017 ESC Guidelines for the management of acute myocardial infarction in patients presenting with ST-segment elevation: the Task Force for the management of acute myocardial infarction in patients presenting with ST-segment elevation of the European Society of Cardiology (ESC). Eur HeartJ. 2018;39(2):119-77.

4. McCarthy CP, Mullins KV, Sidhu SS, Schulman SP, McEvoy JW. The on- and off-target effects of morphine in acute coronary syndrome: a narrative review. Am Heart J. 2016;176:114-21.

5. Kubica J, Adamski P, Ostrowska M, et al. Morphine delays and attenuates ticagrelor exposure and action in patients with myocardial infarction: the randomized, doubleblind, placebo-controlled IMPRESSION trial. Eur Heart J. 2016;37(3):245-52.

6. Parodi G, Bellandi B, Xanthopoulou I, et al. Morphine is associated with a delayed activity of oral antiplatelet agents in patients with ST-elevation acute myocardial infarction undergoing primary percutaneous coronary intervention. Circ Cardiovasc Interv. 2014;8(1):e1593.

7. Tantry US, Bonello L, Aradi D, et al. Consensus and update on the definition of on-treatment platelet reactivity to adenosine diphosphate associated with ischemia and bleeding. JAm Coll Cardiol. 2013;62(24):2261-73.

8. Bonello L, Pansieri M, Mancini J, et al. High on-treatment platelet reactivity after prasugrel loading dose and cardiovascular events after percutaneous coronary intervention in acute coronary syndromes. J Am Coll Cardiol. 2011;58(5):467-73.

9. McEvoyJW, Ibrahim K, Kickler TS, etal. Effect of intravenous fentanyl on ticagrelor absorption and platelet inhibition among patients undergoing percutaneous coronary intervention: the PACIFY randomized clinical trial (Platelet Aggregation With Ticagrelor Inhibition and Fentanyl). Circulation. 2018;137(3):307-9.

10. Silvain J, Storey RF, Cayla G, et al. P2Y12 receptor inhibition and effect of morphine in patients undergoing primary PCI for ST-segment elevation myocardial infarction. The PRIVATE-ATLANTIC study. Thromb Haemost. 2016;116(2):369-78.

11. Hobl EL, Stimpfl T, Ebner J, et al. Morphine decreases clopidogrel concentrations and effects: a randomized, double-blind, placebo-controlled trial. J Am Coll Cardiol. 2014;63(7):630-5.

12. Johnson TW, Mumford AD, Scott LJ, et al. A study of platelet inhibition, using a 'point of care' platelet function test, following primary percutaneous coronary intervention for ST-elevation myocardial infarction [PINPOINT-PPCI.PLoS ONE. 2015;10(12):e144984.

13. Bellandi B, Zocchi C, Xanthopoulou I, et al. Morphine use and myocardial reperfusion in patients with acute myocardial infarction treated with primary PCI. Int J Cardiol. 2016;221:567-71.

14. de Waha S, Eitel I, Desch S, et al. Intravenous morphine administration and reperfusion success in ST-elevation myocardial infarction: insights from cardiac magnetic resonance imaging. Clin Res Cardiol. 2015;104(9):727-34.

15. Meine TJ, Roe MT, Chen AY, etal. Association of intravenous morphine use and outcomes in acute coronary syndromes: results from the CRUSADE Quality Improvement Initiative. AmHeartJ. 2005;149(6):1043-9.

16. Puymirat E, Lamhaut L, Bonnet N, et al. Correlates of prehospital morphine use in ST-elevation myocardial infarction patients and its association with in-hospital outcomes and long-term mortality: the FAST-MI (French Registry of Acute ST-elevation and non-ST-elevation Myocardial Infarction) programme. Eur Heart J. 2016;37(13):1063-71.

17. Parodi G, Xanthopoulou I, Bellandi B, et al. Ticagrelor crushed tablets administration in STEMI patients: the MOJITO study. JAm Coll Cardiol. 2015;65(5):511-2.

18. Niezgoda P, Sikora J, Baranska M, et al. Crushed sublingual versus oral ticagrelor administration strategies in patients with unstable angina. A pharmacokinetic/pharmacodynamic study. Thromb Haemost. 2017;117(4):718-26.

19. Cutlip DE, Nakazawa G, Krucoff MW, et al. Autopsy validation study of the academic research consortium stent thrombosis definition. JACC Cardiovasc Interv. 2011;4(5):554-9.

20. Thygesen K, Alpert JS, Jaffe AS, et al. Third universal definition of myocardial infarction. Eur Heart J. 2012;33(20):2551-67.

21. Mehran R, Rao SV, Bhatt DL, et al. Standardized bleeding definitions for cardiovascular clinical trials: a consensus report from the Bleeding Academic Research Consortium. Circulation. 2011;123(23):2736-47.

22. Martini AK, Rodriguez CM, Cap AP, Martini WZ, Dubick MA. Acetaminophen and meloxicam inhibit platelet aggregation and coagulation in blood samples from humans. Blood Coagul Fibrinolysis. 2014;25(8):831-7.

23. MunsterhjelmE, Munsterhjelm NM, NiemiTT, YlikorkalaO, Neuvonen PJ, Rosenberg PH. Dose-dependentinhibition of platelet function by acetaminophen in healthy volunteers. Anesthesiology. 2005;103(4):712-7.

24. Galliard-Grigioni KS, Fehr M, Reinhart WH. Influence of combinations of acetylsalicylic acid, acetaminophen, and diclofenac on platelet aggregation. Eur J Pharmacol. 2008;595(1-3):65-8.

25. Ibrahim K, Goli RR, Shah R, Resar JR, Schulman SP, McEvoy JW. Effect of intravenous fentanyl on ticagrelor absorption and platelet inhibition among patients undergoing percutaneous coronary intervention: design, rationale, and sample characteristics of the PACIFY randomized trial. Contemp Clin. Trials. 2018;64:8-12.

26. Montalescot G. van't Hof AW, Lapostolle F, et al. Prehospital ticagrelor in ST-segment elevation myocardial infarction. NEngl J Med. 2014;371(11):1016-27.

27. Bhatt DL, Stone GW, Mahaffey KW, et al. Effect of platelet inhibition with cangrelor during PCI on ischemic events. NEngl J Med. 2013;368(14):1303-13.

28. Venetsanos D, Sederholm Lawesson S, Swahn E, Alfredsson J. Chewed ticagrelor tablets provide faster platelet inhibition compared to integral tablets: the inhibition of platelet aggregation after administration of three different ticagrelor formulations (IPAAD-Tica) study, a randomised controlled trial. Thromb Res. 2017;149:88-94.

29. Mukherjee D, Nissen SE, Topol EJ. Risk of cardiovascular events associated with selective COX-2 inhibitors. JAMA. 2001;286(8):954-9.

30. Cantais A, Schnell D, Vincent F, et al. Acetaminopheninduced changes in systemic blood pressure in critically ill patients: results of a multicenter cohort study. Crit Care Med. 2016;44(12):2192-8. 